'División de Enfermedades

Cardiovasculares, Escuela de Medicina, Facultad de Medicina, Pontificia Universidad Católica de Chile.

2División de Salud Pública, Escuela de Medicina, Facultad de Medicina Pontificia Universidad Católica de Chile.

${ }^{3}$ Departamento de

Matemáticas, Universidad de la Serena.

${ }^{4}$ Departamento de Nefrología, Escuela de Medicina, Facultad de

Medicina, Pontificia

Universidad Católica de Chile.

${ }^{a}$ Enfermera Matrona.

Recibido el 1 de febrero de 2013, aceptado el 12 de junio de 2013

Correspondencia a: Dra. Mónica Acevedo Escuela de Medicina, Facultad de Medicina, División de Enfermedades Cardiovasculares, Pontificia Universidad Católica de Chile, Santiago, Chile. Lira \# 85, Primer Piso, Santiago, Chile.

Teléfono: 5623543334

E-mail:macevedo@med. puc.cl

\section{¿Sirven los marcadores de riesgo no tradicionales en la diferenciación del riesgo cardiovascular en adultos jóvenes con exceso de peso?}

\author{
VERÓNICA KRÄMER ${ }^{1, a}$, RAMÓN CORBALÁN ${ }^{1}$, XIMENA BERRÍOS ${ }^{2}$, \\ CARLOS NAVARRETE ${ }^{3}$, RODRIGO TAGLE ${ }^{4}$, MÓNICA ACEVEDO ${ }^{1}$
}

\section{Carotid intima media thickness and $\mathrm{C}$ reactive protein among overweight or obese subjects without metabolic syndrome}

Background: Recognizing cardiovascular risk in overweight adults is challenging, as they usually have a low Framingham risk score (FRAM). In these subjects, non-traditional biomarkers could improve risk stratification. Aim: To assess carotid intima media thickness (CIMT) and ultrasensitive C-reactive protein (usCRP) among overweight and obese subjects without metabolic syndrome (MetSyn). Subjects and Methods: In 1558 asymptomatic participants (816 women, $45 \pm 11$ years) we measured body mass index (BMI), waist circumference, blood pressure, lipid profile, blood glucose, FRAM, usCRP and CIMT. For analytical purposes, we divided the subjects in three groups according to BMI and number of ATPIII-MetSyn risk factors (RF): 1) $B M I<25$ and $<3 R F, 2) B M I \geq 25$ and $<3 R F$ and 3) $B M I \geq 25$ and $\geq 3 R F$. Results: Participants of group 2 (BMI $\geq 25$ and $<3 R F)$ had a low FRAM (8\%). Compared with participants of group 1, they had a higher CIMT $(0.61 \pm 0.1$ and $0.57 \pm 0.09 \mathrm{~mm}$, respectively, $p<0.01)$ and $u s C R P(2.1 \pm 2.1$ and $1.5 \pm 1.9 \mathrm{mg} / \mathrm{L}$ respectively, $p<0.01)$. Conclusions: This study shows that although subjects with overweight/obesity without MetSyn have low cardiovascular risk based on FRAM, they have higher CIMT and usCRP than their normal weight counterparts.

(Rev Med Chile 2013; 141: 1026-1033).

Key words: Carotid Intima-media thickness; Metabolic syndrome X; Obesity; Risk factors.

\section{A} ctualmente, en nuestro país existe una epidemia de sobrepeso y obesidad con prevalencias de $39 \%$ y $25 \%$, respectivamente ${ }^{1}$. El gran problema es que el riesgo cardiovascular (CV) en sujetos con exceso de peso es heterogéneo y los estudios han sido confundentes: algunos han mostrado que los sujetos "sobrepeso metabólicamente sanos", es decir, que no presentan factores de riesgo (FR) metabólicos, pero que tienen exceso de peso, presentan más riesgo $\mathrm{CV}$ y/o aterosclerosis subclínica que los sujetos "normopeso metabólicamente sanos"2-4. Un análisis reciente demostró que los sujetos obesos tenían un riesgo CV 40 a $200 \%$ mayor $^{5}$. Es por ello que se ha sugerido que estos individuos sí tienen riesgo $\mathrm{CV}$, pero que se demora en evidenciarse clínicamente. Por otro lado, existen estudios que muestran que los sujetos con exceso de peso y metabólicamente sanos tienen menos riesgo CV medido por eventos ${ }^{6}$. Ortega y cols ${ }^{6}$ demostraron que la condición de "obesos metabólicamente sanos" era una condición "benigna", con un mejor pronóstico CV que los obesos metabólicamente enfermos.

El otro problema es que los adultos con exceso 
Biomarcadores y riesgo cardiovascular en sujetos con exceso de peso - V. Krämer et al

de peso, generalmente, presentan un puntaje de Framingham (FRAM) bajo, y por ello, son considerados "bajo riesgo" y no son intervenidos.

Para esto se han investigado nuevos FR CV, que ayudan a una mejor estratificación del riesgo de los pacientes habitualmente subestimados, como los sujetos con exceso de peso, entre otros.

Entre los FR no tradicionales más estudiados están la proteína C-reactiva ultrasensible (PCRus) y el grosor íntima medio carotideo (IMT). La PCRus es un marcador de riesgo de eventos ateroescleróticos coronarios y cerebrovasculares. También se relaciona estrechamente a obesidad visceral y es un marcador de riesgo metabólico de diabetes $^{7-11}$. Por su parte, el IMT es un marcador de aterosclerosis subclínica, que también predice eventos ateroescleróticos ${ }^{12-15}$.

La hipótesis que nos planteamos fue la siguiente: Las personas con exceso de peso "metabólicamente sanas" tienen mayor riesgo CV basado en dos biomarcadores no tradicionales, PCRus e IMT, que los sujetos normopeso "metabólicamente sanos".

\section{Material y Método}

Estudio transversal estratificado en hombres y mujeres de 25 a 64 años de la población del estudio CARMELA-Chile ${ }^{16}$. Se excluyeron sujetos con antecedente de enfermedad aterosclerótica, infección reciente, enfermedades inflamatorias no infecciosas conocidas, o con PCRus $>10 \mathrm{mg} / \mathrm{L}$.

\section{Recolección de datos}

Los sujetos fueron encuestados sobre antecedentes demográficos y médicos, FR CV y medicamentos. Luego, se les midió peso, talla, índice de masa corporal (IMC), cintura, cadera, y presión $\operatorname{arterial}^{17}$, y se realizó perfil lipídico, glicemia, PCRus e IMT.

En todos los sujetos se calculó FRAM, según Wilson y cols $^{18}$, como riesgo de infarto o muerte a 10 años.

\section{Mediciones de laboratorio}

Las muestras fueron obtenidas por punción venosa, con ayuno de $12 \mathrm{~h}$ y procesadas por los siguientes métodos:

- Colesterol total, HDL y triglicéridos: métodos enzimáticos estándar con reactivos ad-hoc (analizador Hitachi).
- Colesterol LDL: calculado por fórmula de Friedewald.

- Glicemia: método glucosa oxidasa.

\section{PCR ultrasensible:}

La PCRus fue medida usando método nefelométrico (Dade Behring BN II; límite de detección 0,1 mg/L).

Para el análisis de los resultados, se la consideró como variable continua y también, de forma dicotómica, con un corte de $<2 \mathrm{mg} / \mathrm{L}$ como normal ${ }^{13}$.

\section{Medición de grosor intima-media carotídeo (IMT)}

Se realizó según las recomendaciones del consenso de Mannheim ${ }^{19}$, utilizando un transductor lineal de alta frecuencia (5-13 Mhz). La imagen se focalizó en la pared posterior de cada carótida común, en áreas sin placas, donde se eligió un segmento de $1 \mathrm{~cm}$, proximal a la bifurcación carotidea de cada lado. Se realizaron mediciones en cada carótida común, en 3 ventanas ecográficas, utilizando un software con medición automática de bordes. Todas las mediciones se realizaron "offline" y en fin de diástole. El coeficiente de variación intraoperador de nuestro grupo es de 3,8\%. Los valores percentilados de IMT en Santiago han sido previamente publicados ${ }^{20}$.

Para efectos de análisis estadístico, se utilizó el IMT medio máximo. Placa carotídea se definió como una protrusión en el lumen mayor a 50\% del IMT adyacente o IMT $>1,5 \mathrm{~mm}$. Aterosclerosis subclínica fue definida como presencia de placa carotidea y/o IMT sobre el percentil 75.

\section{Definiciones de grupos, de acuerdo al estado nutricional (IMC) y FR metabólico}

Para el análisis, la muestra fue dividida de acuerdo al estado nutricional (IMC) y a la agregación de FR de síndrome metabólico (SMET), utilizando la definición clásica de SMET por ATPIII ${ }^{21}$ :

- Presión arterial sistólica $\geq 130 \mathrm{mmHg}$ y/o diastólica $\geq 85 \mathrm{mmHg}$.

- Cintura $>88 \mathrm{~cm}$ en mujeres o $>102 \mathrm{~cm}$ en hombres.

- $\mathrm{HDL}<50 \mathrm{mg} / \mathrm{dL}$ en mujeres o $<40 \mathrm{mg} / \mathrm{dL}$ en hombres.

- Glicemia $\geq 100 \mathrm{mg} / \mathrm{dl}$ o uso de medicación para la diabetes.

- Triglicéridos $\geq 150 \mathrm{mg} / \mathrm{dl}$ o uso de tratamiento hipolipemiante. 
Biomarcadores y riesgo cardiovascular en sujetos con exceso de peso - V. Krämer et al

Así, se crearon cuatro grupos diferentes de sujetos:

- Normopeso "metabólicamente sanos" = IMC $<25$ y $<3$ FR $(\mathrm{n}=391)$.

- Normopeso "metabólicamente enfermos" = IMC $<25 \mathrm{y} \geq 3$ FR $(\mathrm{n}=64)$.

- Sobrepeso "metabólicamente sanos" = IMC $\geq 25$ $\mathrm{y}<3$ FR $(\mathrm{n}=509)$.

- Sobrepeso "metabólicamente enfermos" = IMC $\geq 25 \mathrm{y} \geq 3$ FR $(\mathrm{n}=594)$.

Los grupos objetivos de comparación de este estudio fueron los sujetos normopeso "metabólicamente sanos" versus los sobrepeso "metabólicamente sanos".

Debido a que el grupo de sujetos normopeso "metabólicamente enfermo" era muy pequeño (64 personas), se excluyó de la presentación de los resultados.

\section{Análisis estadístico}

Los resultados se presentan como media \pm D.E o frecuencia (\%) según corresponda. Para las comparaciones de medias se ocupó análisis de varianza y regresión lineal. Para las comparaciones de proporciones se ocupó test exacto de Fisher. La PCRus se analizó en escala logarítmica para corregir asimetría de distribución.
Para todo el análisis estadístico se ocupó software R 2.15.

\section{Aspectos éticos}

Todos los sujetos firmaron el consentimiento informado del estudio, aprobado por el comité de ética de la universidad ejecutora.

\section{Resultados}

Se incluyeron 1.558 sujetos (edad promedio 45 $\pm 11,52 \%$ mujeres). Cuarenta y cinco por ciento era fumador, $89 \%$ sedentario, $12 \%$ diabético, $70 \%$ dislipidémico, $31 \%$ hipertenso y $18 \%$ tenía historia familiar de cardiopatía coronaria.

En la Tabla 1 se muestran los valores promedio de los componentes del SMET, FRAM, PCRus, valores de IMT y prevalencia de aterosclerosis subclínica en la muestra total y por sexo.

En la Tabla 2 se presentan los grupos divididos según estado nutricional (IMC) y agregación de FR de SMET y se comparan los valores promedio de HDL, triglicéridos, glicemia, presión arterial, cintura, FRAM, PCRus, IMT y prevalencia de aterosclerosis subclínica. Como se aprecia en la Tabla 2, y como era esperable, los sujetos "sobrepeso metabólicamente enfermos" presentaron en for-

Tabla 1. Valores promedio de índice de masa corporal, componentes del síndrome metabólico, Puntaje de Framingham, proteína C-reactiva ultrasensible, valores de íntima media carotideo y prevalencia de aterosclerosis subclínica en la muestra total y dividida por sexo

\begin{tabular}{|c|c|c|c|}
\hline & $\begin{array}{c}\text { Total } \\
(\mathrm{n}=1.558)\end{array}$ & $\begin{array}{l}\text { Hombres } \\
(n=741)\end{array}$ & $\begin{array}{c}\text { Mujeres } \\
(n=816)\end{array}$ \\
\hline IMC (kg./m²) & $28 \pm 5$ & $28 \pm 4$ & $28 \pm 6$ \\
\hline Colesterol HDL (mg/dL) & $50 \pm 13$ & $46 \pm 12$ & $53 \pm 13$ \\
\hline Triglicéridos (mg/L) & $156 \pm 106$ & $174 \pm 114$ & $139 \pm 96$ \\
\hline Glicemia (mg/dL) & $101 \pm 37$ & $99 \pm 41$ & $103 \pm 34$ \\
\hline Presión arterial sistólica (mmHg) & $120 \pm 20$ & $123 \pm 19$ & $118 \pm 21$ \\
\hline Presión arterial diastólica $(\mathrm{mmHg})$ & $78 \pm 12$ & $82 \pm 12$ & $76 \pm 12$ \\
\hline Cintura (cm) & $91 \pm 11$ & $94 \pm 10$ & $88 \pm 12$ \\
\hline Puntaje de Framingham (\%) & $9 \pm 9$ & $13 \pm 10$ & $7 \pm 7$ \\
\hline PCRus (mg/L) & $2,3 \pm 2,3$ & $1,9 \pm 2$ & $2,6 \pm 2,4$ \\
\hline IMT medio máximo (mm) & $0,61 \pm 0,11$ & $0,63 \pm 0,11$ & $0,61 \pm 0,11$ \\
\hline Placa carotídea o IMT > 75 (\%) & 21 & 24 & 18 \\
\hline
\end{tabular}

$\mathrm{IMC}=$ Índice de Masa Corporal; PCRus= Proteína C-Reactiva Ultrasensible; IMT= Grosor Íntima Media Carotideo Pc= Percentil. 
Tabla 2. Comparación de grupos divididos según estado nutricional y agregación de factores de riesgo de síndrome metabólico: valores promedio de HDL, triglicéridos, glicemia, presión arterial, cintura, Puntaje de Framingham, proteína C-reactiva ultrasensible, íntima media carotideo y prevalencia de aterosclerosis subclínica

\begin{tabular}{|c|c|c|c|c|c|}
\hline & $\begin{array}{c}\text { Normopesos } \\
\text { "metabólicamente } \\
\text { sanos" } \\
\text { IMC }<25 \text { y < } 3 \text { FR }\end{array}$ & $\begin{array}{c}\text { Sobrepeso } \\
\text { "metabólicamente } \\
\text { sanos" } \\
\text { IMC } \geq 25 \text { y < } 3 \text { FR }\end{array}$ & $\mathbf{p}^{\dagger}$ & $\begin{array}{c}\text { Sobrepeso } \\
\text { "metabólicamente } \\
\text { enfermos" } \\
\text { IMC } \geq 25 \text { y } \geq 3 \text { FR }\end{array}$ & $\mathbf{p}^{ \pm}$ \\
\hline Edad (años) & $41 \pm 11$ & $44 \pm 11$ & $<0,0001$ & $47 \pm 11$ & $<0,0001$ \\
\hline IMC $\left(\mathrm{kg} / \mathrm{m}^{2}\right)$ & $23 \pm 2$ & $28 \pm 3$ & $<0,0001$ & $31 \pm 4$ & $<0.0001$ \\
\hline $\mathrm{HDL}(\mathrm{mg} / \mathrm{dL})$ & $56 \pm 13$ & $52 \pm 12$ & $<0,0001$ & $44 \pm 11$ & $<0,0001$ \\
\hline TG (mg/dL) & $98 \pm 52$ & $124 \pm 72$ & $<0,0001$ & $215 \pm 123$ & $<0,0001$ \\
\hline GLIC (mg/dL) & $97 \pm 41$ & $101 \pm 39$ & $<0,001$ & $105 \pm 30$ & 0,02 \\
\hline PAS (mmHg) & $110 \pm 17$ & $117 \pm 16$ & $<0,0001$ & $130 \pm 21$ & $<0,0001$ \\
\hline PAD $(\mathrm{mmHg})$ & $72 \pm 11$ & $77 \pm 10$ & $<0,0001$ & $84 \pm 13$ & $<0,0001$ \\
\hline Cintura (cm) & $80 \pm 7$ & $92 \pm 8$ & $<0,0001$ & $99 \pm 10$ & $<0,0001$ \\
\hline FRAM (\%) & $5 \pm 5$ & $8 \pm 7$ & $<0,0001$ & $13 \pm 10$ & $<0,0001$ \\
\hline PCRus (mg/L) & $1,5 \pm 1,9$ & $2,1 \pm 2,1$ & $<0,0001$ & $3,0 \pm 2,4$ & $<0,0001$ \\
\hline PCRus $>2 \mathrm{mg} / \mathrm{L}$ & 19 & 32 & $<0,001$ & 53 & $<0,0001$ \\
\hline IMT (mm) & $0,57 \pm 0,09$ & $0,61 \pm 0,1$ & $<0,0001$ & $0,64 \pm 0,11$ & $<0,0001$ \\
\hline ATE subclínica (\%) & 12 & 19 & $<0,01$ & 27 & $<0,0001$ \\
\hline
\end{tabular}

$\mathrm{IMC}=$ Índice de Masa Corporal; TG = Triglicéridos; $\mathrm{GLIC}=$ Glicemia; PAS/PAD = presión arterial sistólica/diastólica; FRAM= Puntaje de Framingham; PCRus= Proteína C-Reactiva Ultrasensible; IMT= Grosor Íntima Media Carotideo; ATE subclínica= aterosclerosis subclínica definida como placa carotídea o IMT > Pc75 (\%). p $^{\dagger}=$ p entre Normopesos "metabólicamente sanos" y Sobrepeso "metabólicamente sanos". $\mathrm{p}^{\ddagger}=\mathrm{p}$ entre los tres grupos.

ma significativa, un perfil de riesgo más deletéreo que los otros dos grupos. En la comparación de los grupos objetivo: sujetos "normopeso metabólicamente sanos" y "sobrepeso metabólicamente sanos", se demuestra que los sobrepeso, a pesar de tener $<3$ FR de SMET, tenían niveles significativamente mayores de triglicéridos $(\mathrm{p}<0,0001)$, glicemia $(\mathrm{p}<0,001)$, presión arterial sistólica y diastólica $(\mathrm{p}<0,0001)$ y cintura $(\mathrm{p}<0,0001)$. El colesterol HDL de los sujetos sobrepeso era también significativamente menor que el de los normopeso $(\mathrm{p}<0,0001)$.

El FRAM en los "sobrepeso metabólicamente sanos" y los "normopeso metabólicamente sanos" fue de riesgo bajo ( 8 y $5 \%$ respectivamente).

Con respecto a la PCRus, los sujetos "sobrepeso metabólicamente sanos", tuvieron valores significativamente más altos que los "normopeso metabólicamente sanos": 2,1 versus $1,5 \mathrm{mg} / \mathrm{L}$ $(\mathrm{p}<0,0001)$ (Tabla 2 y Figura 1). Además, destaca

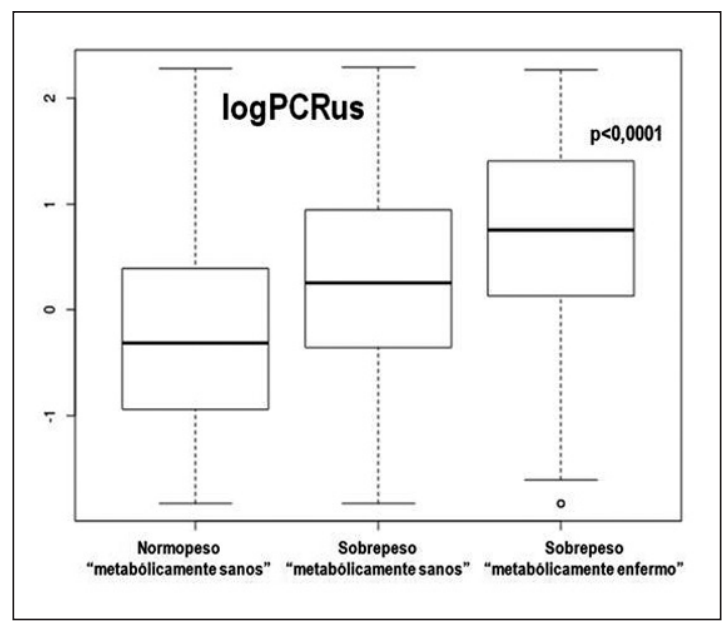

Figura 1. Valores promedio de Proteína C-Reactiva ultrasensible según composición corporal (IMC) y agregación de factores de riesgo de síndrome metabólico según criterio ATPIII, ajustados por edad, sexo y actividad física. ${ }^{*} \log P C R u s=$ Logaritmo de Proteína C-Reactiva Ultrasensible. 
que $32 \%$ de los sujetos "sobrepeso metabólicamente sanos" tuvo la PCRus elevada ( $>2 \mathrm{mg} / \mathrm{L})$, a diferencia de $19 \%$ del grupo normopeso $(\mathrm{p}<0,001)$.

Del mismo modo, los sujetos "sobrepeso metabólicamente sanos" presentaron un IMT significativamente más alto que los "normopeso metabólicamente sanos": 0,61 versus $0,57 \mathrm{~mm}$ respectivamente $(\mathrm{p}<0,0001)$ (Tabla 2 y Figura 2$)$. Además, los sujetos "sobrepeso metabólicamente sanos" presentaron en forma significativa, más aterosclerosis subclínica, definida como presencia de placa carotidea y/o IMT sobre el percentil 75, que los sujetos "normopeso metabólicamente sanos": 19 versus $12 \%(\mathrm{p}<0,01)$ (Tabla 2$)$ respectivamente. Así, a pesar de que los sujetos "sobrepeso metabólicamente sanos" tuvieron un FRAM bajo (8\%), presentaron significativamente mayor riesgo basado en PCRus y aterosclerosis subclínica, que los "normopeso metabólicamente sanos".

\section{Discusión}

En este estudio demostramos que el grupo de sujetos sobrepeso "metabólicamente sanos", si bien tiene bajo riesgo CV según FRAM, presenta importante carga inflamatoria y de aterosclerosis subclínica, en comparación a sus pares normopesos "metabólicamente sanos". Estos resultados enfatizan la necesidad de considerar estos individuos sobrepeso "metabólicamente sanos", como un grupo "en riesgo CV”. En nuestro país este grupo es muy prevalente, y no está incluido actualmente en el programa de salud CV.

En clínica, el problema cuando hablamos de sujetos con exceso de peso, es que su riesgo CV es heterogéneo: algunos autores han reportado que tienen bajo riesgo futuro, es decir, una condición "benigna", mientras que otros, afirman que sí tienen riesgo, pero que no es inmediato, sino que se manifiesta en años.

Así Ortega y cols $^{6}$, recientemente, reportaron que los obesos "metabólicamente sanos" tienen mejor pronóstico $\mathrm{CV}$ que los sujetos obesos "metabólicamente enfermos", pero únicamente después de ajustar por capacidad aeróbica: los sujetos obesos "metabólicamente sanos" de su estudio presentaban un mejor acondicionamiento aeróbico que los obesos "metabólicamente enfermos", y sólo después de considerar ese factor, su morbimortalidad era más baja.

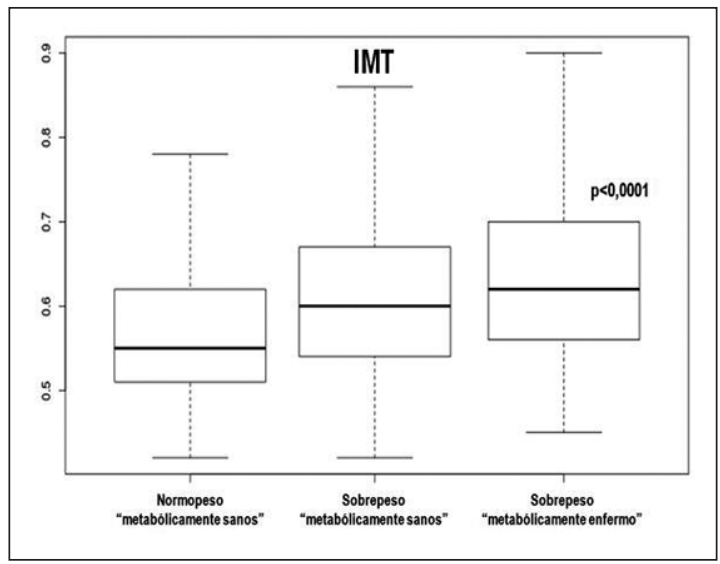

Figura 2. Valores promedio de Grosor Íntima Media Carotideo según composición corporal (IMC) y agregación de factores de riesgo de síndrome metabólico según criterio ATPIII, ajustados por edad, sexo y actividad física. ${ }^{*} \mathrm{IMT}=$ Grosor Íntima Media Carotideo.

En contraste, Marini y cols ${ }^{3}$ reportaron en mujeres obesas "metabólicamente sanas", que el grosor carotideo era intermedio entre las normopeso "metabólicamente sanas" y la obesas "metabólicamente enfermas". Stefan y cols ${ }^{4}$ reportaron algo similar: los sujetos obesos, pero sensibles a la insulina, presentaron un IMT mayor que los controles normopeso "metabólicamente sanos". Finalmente, un subanálisis de los estudios Health Profesional's Follow-up Study y Nurse's Health Study demostró que los sujetos obesos "sanos" tenían un riesgo CV 40 a 200\% mayor, que los normpesos sanos, pero que este riesgo demoraba en evidenciarse ${ }^{5}$. En nuestro estudio, demostramos que la condición de exceso de peso "metabólicamente sana" no es benigna, incluso después de ajustar por varios confundentes, como edad, sexo y actividad física. Así, nuestros resultados apoyan esta última evidencia, es decir, que los sujetos con exceso peso, aunque sean "metabólicamente sanos", presentan más riesgo $\mathrm{CV}$.

El SMET, como entidad clínica, ha sido muy controvertida, ya que la discusión se ha enfocado más en definir si realmente existe desde un punto de vista fisiopatológico, más que en su utilidad clínica en el mundo real, que es orientar y motivar al clínico en la intervención precoz de estos grupos en riesgo, para así evitar el desarrollo futuro de enfermedad CV y diabetes. Previamente, hemos publicado que los sujetos adultos con SMET e 
inflamación tienen mucho más riesgo de aterosclerosis subclínica que los sujetos sólo con SMET ${ }^{22}$. En ese estudio, la presencia de inflamación daba más riesgo de aterosclerosis subclínica dentro del propio grupo de pacientes con SMET. También demostramos que en niños con exceso de peso, que presentan agregación de FR metabólicos, pero que aún no cumplen criterios para SMET, presentan más inflamación (determinada por PCRus) y aterosclerosis subclínica ${ }^{23}$. Si niños tan pequeños como los descritos presentaron más aterosclerosis subclínica, era probable que en los adultos se diera lo mismo. Por esto, nos planteamos la hipótesis de que aquellos adultos con exceso de peso, pero "metabólicamente sanos" tendrían mayor aterosclerosis subclínica.

La última Encuesta Nacional de Salud ${ }^{1}$ reportó un porcentaje muy importante de sujetos con exceso de peso en Chile, casi 65\%. En este sentido, el gran problema en clínica es que FRAM subestima el riesgo CV real en algunas personas, como en los sujetos con exceso de peso y jóvenes, como los de esta muestra. Al subestimar el riesgo, estas personas no son intervenidas en forma agresiva en la etapa preventiva. La adición de dos marcadores de riesgo, PCRus e $\mathrm{IMT}^{13}$ en este estudio, nos ha permitido evidenciar que el exceso de peso es importante por sí solo, y que determina mayor riesgo $\mathrm{CV}$.

La PCRus es el marcador de riesgo no tradicional más usado en la actualidad, contando con un nivel de evidencia IIa para su uso en sujetos asintomáticos ${ }^{13}$. Es un buen marcador de eventos ateroescleróticos y diabetes en población sana y en población con antecedentes $\mathrm{CV}$ previos ${ }^{8}$. Por ello, el hallazgo de PCRus elevada en nuestros sujetos sobrepeso "metabólicamente sanos", clasifica a este grupo como personas con riesgo CV y de diabetes a futuro. Desde el punto de vista clínico, estos sujetos debieran intervenirse y seguirse, con modificación agresiva del estilo de vida, haciendo énfasis en la reducción de peso y actividad física. Un ejemplo de que estas intervenciones funcionan, es el estudio Diabetes Prevention Program ${ }^{24}$, que demostró, en adultos no diabéticos, que la modificación del estilo de vida reducía más el riesgo de diabetes que la metformina. Con respecto a la disminución de eventos CV, el estudio JUPITER ${ }^{25}$ demostró que aquellos sujetos que reducían sus niveles de PCRus con estatinas, presentaron menos riesgo de eventos futuros, independiente de la reducción del LDL. Así, deberá demostrarse en estudios prospectivos, si la intervención de los sujetos con exceso de peso "metabólicamente sanos" e inflamados, reduce los eventos ateroescleróticos en el largo plazo.

Con respecto a la aterosclerosis subclínica existe importante evidencia que la asocia a la enfermedad cardio y cerebrovascular ${ }^{12,15}$. AHA/ ACC recomiendan el uso del IMT y detección de placa carotidea, con indicación IIa en sujetos asintomáticos ${ }^{13}$. Recientemente, se ha publicado un estudio sobre el uso apropiado de IMT en la práctica clínica ${ }^{14}$, proponiéndolo como adecuado en algunos subgrupos de pacientes. En nuestro estudio es evidente la mayor prevalencia de aterosclerosis subclínica en los sujetos sobrepeso "metabólicamente sanos" versus los "normopeso sanos". Estos dos grupos (tabla 2), si bien por definición sólo difieren en el corte de IMC $(<25$ versus $\geq 25$ ), tienen diferencias significativas en todos las variables medidas. De nuevo, desde la visión preventiva, estas personas debieran intervenirse, ya que si bien aún no califican en los niveles de corte para algunos FR, sí calificarán a futuro. El IMT y la presencia de placa podrían ayudar en la selección de estos pacientes.

Finalmente, es importante preguntarse por qué los sujetos con exceso de peso "metabólicamente sanos" presentan mayor riesgo de inflamación y de aterosclerosis. En este sentido, no existe una sola respuesta. El exceso de peso condiciona alteraciones en numerosas vías fisiopatológicas que pudieran estar relacionadas al desarrollo de aterosclerosis $^{26}$. Una de las más importantes es la obesidad visceral, que produce resistencia a la insulina, inflamación, dislipidemia aterogénica e hipertensión arterial, todos factores causales de aterosclerosis. La resistencia a la insulina y la inflamación aumentan también el riesgo de diabetes ${ }^{11}$. El desarrollo de aterosclerosis se asocia a los mismos factores. Després y cols ${ }^{26}$ han planteado que es la cantidad y distribución de la grasa corporal, la que determina el riesgo $\mathrm{CV}$ asociado en estos sujetos, y no sólo el IMC.

De esta manera, el mensaje de este estudio es enfatizar el riesgo subclínico que presentan los sujetos con exceso de peso "metabólicamente sanos". El uso adecuado de estos dos biomarcadores, PCRus e IMT, podría ayudar a diferenciar cuáles deben ser intervenidos.

Nuestro estudio tiene limitaciones: a) estu- 
Biomarcadores y riesgo cardiovascular en sujetos con exceso de peso - V. Krämer et al

dio retrospectivo que no demuestra causalidad; b) reportamos en una población sólo de Santiago urbano; c) consideramos sólo FR de SMET y no otros importantes como determinantes de riesgo $\mathrm{CV}$ (ej. tabaco, antecedente familiar de cardiopatía coronaria); d) no determinados grasa total y visceral por escáner, método de referencia actual para medir distribución de grasa.

\section{Referencias}

1. Gobierno de Chile. Ministerio de Salud. II Encuesta Nacional de Salud C-hmc.

2. Khan UI, Wang D, Thurston RC, Sowers M, SuttonTyrrell K, Matthews KA, et al. Burden of subclinical cardiovascular disease in "metabolically benign" and "at-risk" overweight and obese women: The study of women's health across the nation (SWAN). Atherosclerosis 2011; 217: 179-86.

3. Marini MA, Succurro E, Frontoni S, Hribal ML, Andreozzi F, Lauro R, et al. Metabolically healthy but obese women have an intermediate cardiovascular risk profile between healthy nonobese women and obese insulinresistant women. Diabetes Care 2007; 30: 2145-7.

4. Stefan N, Kantartzis K, Machann J, Schick F, Thamer $\mathrm{C}$, Rittig $\mathrm{K}$ et al. Identification and characterization of metabolically benign obesity in humans. Archives of Internal Medicine 2008; 168: 1609-16.

5. Clinical Guidelines on the identification, evaluation, and treatment of overweight and obesity in adults-the evidence report. National institutes of health. Obesity Research 1998; 6 Suppl 2: 51S-209S.

6. Ortega FB, Lee DC, Katzmarzyk PT, Ruiz JR, Sui X, Church TS et al. The intriguing metabolically healthy but obese phenotype: Cardiovascular prognosis and role of fitness. European Heart Journal 2013; 34: 389-97.

7. Libby P, Ridker PM, Maseri A. Inflammation and atherosclerosis. Circulation 2002; 105: 1135-43.

8. Ridker PM. Clinical application of c-reactive protein for cardiovascular disease detection and prevention. Circulation 2003; 107: 363-9.

9. Ridker PM, Buring JE, Cook NR, Rifai N. C-reactive protein, the metabolic syndrome, and risk of incident cardiovascular events: An 8-year follow-up of 14719 initially healthy american women. Circulation 2003; 107: 391-7.

10. Ridker PM, Hennekens CH, Buring JE, Rifai N. Creactive protein and other markers of inflammation in the prediction of cardiovascular disease in women. The New England Journal of Medicine 2000; 342: 836-43.

11. Han TS, Sattar N, Williams K, González-Villalpando C,
Lean ME, Haffner SM. Prospective study of c-reactive protein in relation to the development of diabetes and metabolic syndrome in the Mexico City diabetes study. Diabetes Care 2002; 25: 2016-21.

12. Lorenz MW, Markus HS, Bots ML, Rosvall M, Sitzer M. Prediction of clinical cardiovascular events with carotid intima-media thickness: A systematic review and metaanalysis. Circulation 2007; 115: 459-67.

13. Greenland P, Alpert JS, Beller GA, Benjamin EJ, Budoff MJ, Fayad ZA, et al. 2010 ACCF/AHA guideline for assessment of cardiovascular risk in asymptomatic adults: A report of the American College of Cardiology Foundation/American Heart Association task force on practice guidelines. Journal of the American College of Cardiology 2010; 56: e50-103.

14. Society of Atherosclerosis Imaging and Prevention Developed in collaboration with the International Atherosclerosis Society. Appropriate use criteria for carotid intima media thickness testing. Atherosclerosis 2011; 214: 43-6.

15. Stein JH, Korcarz CE, Hurst RT, Lonn E, Kendall CB, Mohler ER, et al. Use of carotid ultrasound to identify subclinical vascular disease and evaluate cardiovascular disease risk: A consensus statement from the American Society of Echocardiography carotid intima-media thickness task force. Endorsed by the Society for Vascular Medicine. Journal of the American Society of Echocardiography: official publication of the American Society of Echocardiography 2008; 21: 93-111; quiz 189-90.

16. Schargrodsky H, Hernández-Hernández R, Champagne BM, Silva H, Vinueza R, Silva Aycaguer LC, et al. CARMELA: Assessment of cardiovascular risk in seven latin american cities. Am J Med 2008; 121: 58-65.

17. Chobanian AV, Bakris GL, Black HR, Cushman WC, Green LA, Izzo JL, et al. The seventh report of the joint national committee on prevention, detection, evaluation, and treatment of high blood pressure: The JNC 7 report. JAMA: the Journal of the American Medical Association 2003; 289: 2560-72.

18. Wilson PW, D’Agostino RB, Levy D, Belanger AM, Silbershatz H, Kannel WB. Prediction of coronary heart disease using risk factor categories. Circulation 1998; 97: 1837-47.

19. Touboul PJ, Hennerici MG, Meairs S, Adams H, Amarenco P, Bornstein N, et al. Mannheim carotid intimamedia thickness consensus (2004-2006). An update on behalf of the advisory board of the 3rd and 4th Watching the risk Symposium, 13th and 15th European stroke conferences, Mannheim, Germany, 2004, and Brussels, Belgium, 2006. Cerebrovasc Dis 2007; 23: $75-$ 80.

20. Touboul PJ, Vicaut E, Labreuche J, Acevedo M, Torres 
V, Ramírez-Martínez J, et al. Common carotid artery intima-media thickness: The cardiovascular risk factor multiple evaluation in Latin America (CARMELA) study results. Cerebrovasc Dis 2011; 31: 43-50.

21. Grundy SM, Brewer HB Jr, Cleeman JI, Smith SC Jr, Lenfant C, American Heart A, et al. Definition of metabolic syndrome: Report of the National Heart, Lung, and Blood Institute/American Heart Association conference on scientific issues related to definition. Circulation 2004; 109: 433-8.

22. Acevedo M, Arnaiz P, Corbalán R, Godoy I, Morales D, Chalhub M, et al. Modificación del grosor intima-media carotídeo según factores de riesgo clásicos y síndrome metabólico con o sin inflamación. Rev Chil Cardiol 2009; 28: 337-48.

23. Barja S, Acevedo M, Arnaiz P, Berríos X, Bambs C,
Guzmán B, et al. [Early markers for atherosclerosis and metabolic syndrome in children]. Rev Med Chile 2009; 137: 522-30.

24. Knowler WC, Barrett-Connor E, Fowler SE, Hamman RF, Lachin JM, Walker EA, et al. Reduction in the incidence of type 2 diabetes with lifestyle intervention or metformin. The New England Journal of Medicine 2002; 346: 393-403.

25. Ridker PM, Danielson E, Fonseca FA, Genest J, Gotto AM Jr, Kastelein JJ, et al. Reduction in c-reactive protein and ldl cholesterol and cardiovascular event rates after initiation of rosuvastatin: A prospective study of the JUPITER trial. Lancet 2009; 373: 1175-82.

26. Despres JP. Body fat distribution and risk of cardiovascular disease: An update. Circulation 2012; 126: 1301-13. 